\title{
First order transitions by conduction calorimetry: Application to deuterated potassium dihydrogen phosphate ferroelastic crystal under uniaxial pressure
}

\author{
M. C. Gallardo and J. Jiménez \\ Instituto Mixto Ciencia de Materiales CSIC-Universidad de Sevilla, P.O. Box 1065, Sevilla, Spain \\ M. Koralewski \\ Institute of Physics, A. Mickiewicz University, Umultowska 85, 61-614 Poznan, Poland \\ J. del Cerro ${ }^{a)}$ \\ Instituto Mixto Ciencia de Materiales CSIC-Universidad de Sevilla, P.O. Box 1065, Sevilla, Spain
}

(Received 3 June 1996; accepted for publication 9 December 1996)

\begin{abstract}
The specific heat $c$ and the heat power $W$ exchanged by a Deuterated Potassium Dihydrogen Phosphate ferroelectric-ferroelastic crystal have been measured simultaneously for both decreasing and increasing temperature at a low constant rate $(0.06 \mathrm{~K} / \mathrm{h})$ between 175 and $240 \mathrm{~K}$. The measurements were carried out under controlled uniaxial stresses of 0.3 and $4.5 \pm 0.1$ bar applied to face (110). At $T_{t}=207.9 \mathrm{~K}$, a first order transition is produced with anomalous specific heat behavior in the interval where the transition heat appears. This anomalous behavior is explained in terms of the temperature variation of the heat power during the transition. During cooling, the transition occurs with coexistence of phases, while during heating it seems that metastable states are reached. Excluding data affected by the transition heat, the specific heat behavior agrees with the predictions of a 2-4-6 Landau potential in the range of 4-15 K below $T_{t}$ while logarithmic behavior is obtained in the range from $T_{t}$ to $1 \mathrm{~K}$ below $T_{t}$. Data obtained under 0.3 and 4.5 bar uniaxial stresses exhibit the same behavior. (C) 1997 American Institute of Physics.
\end{abstract}

[S0021-8979(97)05606-5]

\section{INTRODUCTION}

The ferroelectric crystal of potassium dihydrogen phosphate (KDP) shows a well established first order transition at temperature $T_{c}=123 \mathrm{~K}$ as shown by several techniques. ${ }^{1}$ This transition is between a tetragonal paraelectric phase at high temperatures, and an orthorhombic ferroelectricferroelastic phase at low temperatures. The transition is close to tricritical point (TCP) $)^{2}$ and because of this several phenomena show unusual critical behavior with a small latent heat observed. TCP may be easily obtained at hydrostatic pressure about $2.4 \mathrm{kbar}$.

When KDP is deuterated (DKDP crystal), both the transition enthalpy and transition temperature increase ${ }^{3}$ with the degree of deuteration. When KDP is $100 \%$ deuterated, a first order transition occurs at $220 \mathrm{~K}$. The polarization, which is considered the order parameter, the shear strain $u_{x y}$ and the birrefringence $\Delta n_{x y}$ are proportional to each other. This property allows the use of an external shear stress $\sigma_{x y}$ or an external electric field $E_{z}$ to induce modifications in $u_{x y}$ and $P_{z}$. Consequently, a competition ${ }^{4}$ between electrostatic and elastic energies takes place.

DKDP has been studied by means of different techniques ${ }^{1}$ such as neutron diffraction, ${ }^{5} \mathrm{x}$ rays,${ }^{6}$ dielectric measurements, ${ }^{7}$ etc. The specific heat has been measured by several authors. ${ }^{8-11}$ The specific heat shows a very high peak at transition temperature $T_{t}$, and in some cases, ${ }^{8,9}$ a double maximum has been observed. This effect has not been clearly explained.

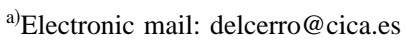

On the other hand, we must point out that:

(i) It is necessary to know when two phases coexist to discriminate data affected by the transition enthalpy, and

(ii) due to the ferroelectric-ferroelastic character of DKDP, the effect of uniaxial pressure and/or the electric field should be known.

In this article the specific heat and the heat power exchanged by a DKDP crystal during the transition were measured simultaneously, under controlled uniaxial pressure. The measurements were made in a conduction calorimeter while cooling or heating the sample at a very low constant rate $(0.06 \mathrm{~K} / \mathrm{h})$.

\section{THE EXPERIMENTAL APPROACH}

The experimental system has previously been described in detail in an earlier work. ${ }^{12}$ The sensor is formed by two fluxmeters $\left(\phi_{1}\right.$ and $\left.\phi_{2}\right)$, two platinum resistance heaters $\left(R_{1}\right.$ and $R_{2}$ ), a calorimeter block (heat sink, $H$ ) and a device $(B)$ to apply an uniaxial pressure to the sample $(S)$. The arrangement of these elements is shown in Fig. 1.

Each fluxmeter is made of 50 chromel-constantan thermocouples connected in series and placed in parallel lines. They are rigid enough to be used to apply uniaxial stress to the crystal. The sample is pressed between the two fluxmeters (Fig. 1). One of them $\left(\phi_{2}\right)$ is fixed to the calorimeter block $(H)$ while the other one $\left(\phi_{1}\right)$ is pressed by bellows $(B)$ connected through a capillary $(C)$ to an outer pressure bottle of $\mathrm{N}_{2}$. An array of valves allows us to control the pressure in 


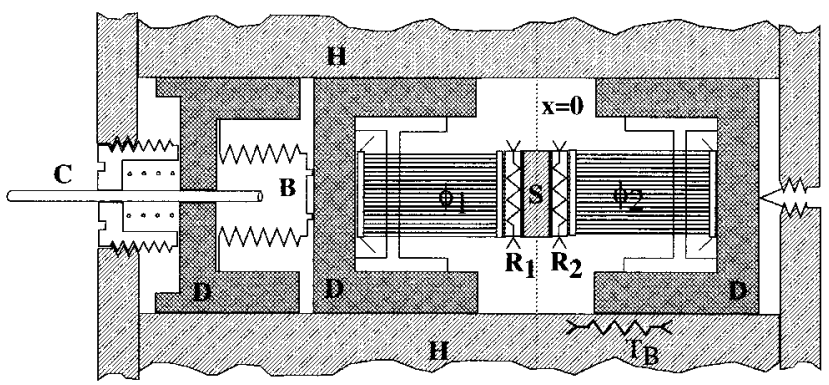

FIG. 1. Diagram of the sensor: $\Phi_{1}$ and $\Phi_{2}$, heat fluxmeters; $R_{1}$ and $R_{2}$, heaters; $S$, sample; $B$, bellows; $D$ fluxmeters and bellows container; $H$, heat sink; $C$, capillary.

the bellows. Extreme precautions were taken to achieve symmetry in the device with respect to plane $x=0$ (shown by vertical dashed line in Fig. 1). The block and two radiation shields surrounding it are placed into a hermetic outer case where a high vacuum can be produced $\left(10^{-7}\right.$ Torr).

The assembly, surrounded by a coiled tube, is placed in a Dewar filled with alcohol. The temperature of the alcohol bath is controlled by a flow of liquid $\mathrm{N}_{2}$ through the coil. The block temperature is measured with a platinum thermometer Leads \& Northrup (mod. 8164 B) and a Tinsley resistance bridge (mod. Ambassador) to $\pm 0.01 \mathrm{~K}$. The emf produced by the fluxmeters is measured by a Keithley 181 nanovoltmeter. All of the devices are controlled by an HP-75000 data acquisition system and a HP-Vectra computer.

Deuterated KDP single crystals were grown by the Holden slow-cooling method. ${ }^{13}$ Very good optical quality crystals were obtained. The degree of deuteration was estimated to be $82 \%$ using the relation between $T_{c}$ and the deuteration content given by Brezina. ${ }^{3}$ The direction perpendicular to (110) face was oriented through the morphology of the crystal and the sample in the form of a cylinder $(3.02 \mathrm{~mm}$ thick and $10.5 \mathrm{~mm}$ in diameter) was prepared. The base of the cylinder coincides with (110) face which allows application of uniaxial pressure $\sigma_{x y}$ to the sample.

The specific heat of the sample is measured using the following procedure: we start from the steady state obtained when the same power $W_{0}$ is dissipated in both heaters $\left(R_{1}\right.$ and $R_{2}$ ). Because there is a high vacuum in the calorimeter $\left(10^{-7}\right.$ Torr) and the maximum temperature difference between sample and block is always less than $0.06 \mathrm{~K}$, we assume there is no lateral heat loss. Thus, $W_{0}$ crosses through the fluxmeter producing a constant emf $V_{0}$. At the initial time the power is cut off and the emf $V(t)$ is integrated up to the time $t_{1}$ when the new thermal equilibrium with the block is reached. Let $V_{1}$ be the constant value of the final emf.

It has been shown ${ }^{14}$ that the thermal capacity of the sample is obtained by

$$
C=\frac{2}{\beta}\left(A-A_{0}\right) \quad A=\int_{0}^{t_{1}} \frac{V(t)-V_{1}}{V_{0}-V_{1}} d t,
$$

where $\beta$ is the thermal resistance of the fluxmeters and $A_{0}$ is the value of $A$ when the experiment is carried out without a sample. $A_{0}$ and $\beta$ are determined by calibration.

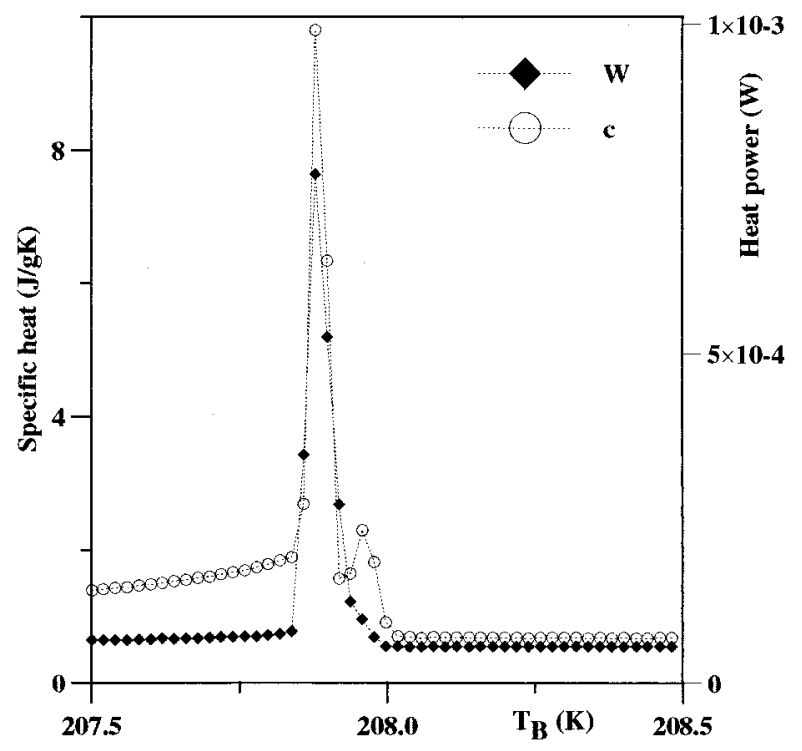

FIG. 2. DKDP specific heat $(\bigcirc)$ and heat power $(\diamond)$ vs block temperature during cooling.

If the measurements are carried out under quasistatic conditions by changing the temperature of the blocks at a very low constant rate (in these measurements $\partial T / \partial t=0.06$ $\mathrm{K} / \mathrm{h}$ ), the emf $V_{1}$ is very small and practically constant. The emf is proportional to the heat flux, which changes the temperature of the sample at the same rate as the temperature of the block ( $V_{1}=\alpha W, \alpha$ determined by calibration). When there is a dissipative effect in the sample or a first order transition is produced, $V_{1}$ changes with time and its value is proportional respectively to the dissipative power or the heat power necessary to provoke the enthalpy change in the transition. Thus, the variation of $V_{1}$ allows us to determine when there is coexistence of phases.

The measurements were carried out for both decreasing and increasing temperature and at two values of applied uniaxial stress on face (110): 0.3 bar and $4.5 \pm 0.1$ bar. The variation of the sample temperature during the measurement process was estimated to be approximately $0.03 \mathrm{~K}$.

\section{RESULTS AND DISCUSSION}

In Fig. 2 the specific heat $c$ and the heat power $W=V_{1} / \alpha$ are represented versus temperature $T_{B}$ of the calorimeter block when cooling and with the uniaxial pressure of 0.3 bar. The data collected in the range $207.85-208.00 \mathrm{~K}$ cover the phase transition point. In this range $c$ shows a double maximum as found in previous ${ }^{8,9}$ measurements. We will attempt to explain this behavior later.

On the other hand, since the temperature difference between the ends of the fluxmeters is $\Delta T=\beta W$, and assuming that the block has a uniform temperature $T_{B}$, the temperature of the sample boundary $T_{S}$ can be obtained. In Fig. 3, $T_{B}$ and $T_{S}$ are represented versus time. Far from $T_{t}$, the difference $T_{S}-T_{B}$ is about $10^{-3} \mathrm{~K}$ and is constant. During the transition, $T_{S}$ seems to remain constant $(207.93 \mathrm{~K})$ like a first order transition with both phases at equilibrium. 


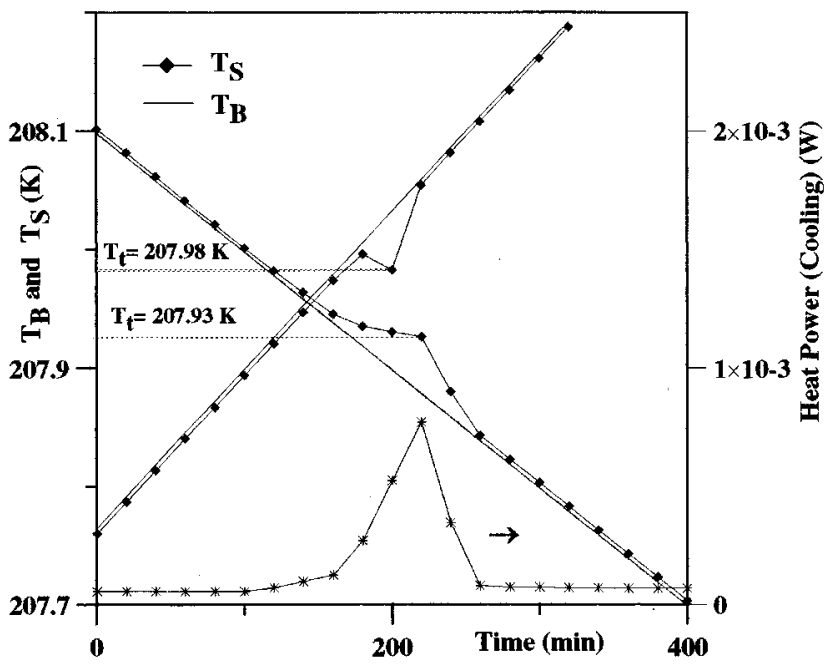

FIG. 3. Block temperature $T_{B}$, and sample boundary temperature $T_{S}$ vs time during cooling and heating. Heat power versus time during cooling.

Thus data obtained for block temperature in the range 207.85-208.00 K are affected by the latent heat. Excluding these data the monophase specific heat of DKDP is represented versus $T_{S}$ in Fig. 4.

To study the behavior of the singular part of the specific heat it is necessary to determine the lattice contribution (base line). We considered three expressions used in the literature ${ }^{15,16}$ to determine the base line: (1) $c_{0}=a+b T$, (2) $c_{0}=a+b T+d T^{2}$, and (3) $c_{0}=a+b / T$, where $a, b, d$ are constants. The best fit for our data is the second expression (where $a=-0.1714, b=5.26 \times 10^{-3}$, and $d=-6.722 \times 10^{-6}$ ), which is represented by the thin line in Fig. 4. It must be pointed out that expressions (2) and (3) practically coincide above $190 \mathrm{~K}$, which is the temperature range we will discuss below.

Figure 4 shows a tail shape behavior of $10 \mathrm{~K}$ above $T_{t}$ similar to that obtained by Reese. ${ }^{10}$ This tail is due to fluctuations of the order parameter. The ferroelectric fluctuations above $T_{t}$ were observed by Bleif et al. ${ }^{17}$ and they correlated

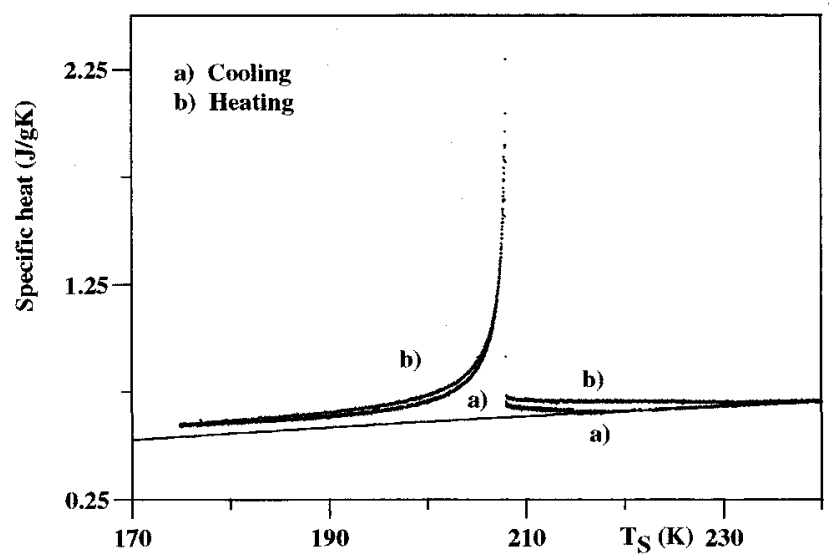

FIG. 4. DKDP specific heat vs sample temperature during cooling (a) and during heating (b) Thin line is best fit by equation of type $c_{0}=a+b T+d T^{2}$.

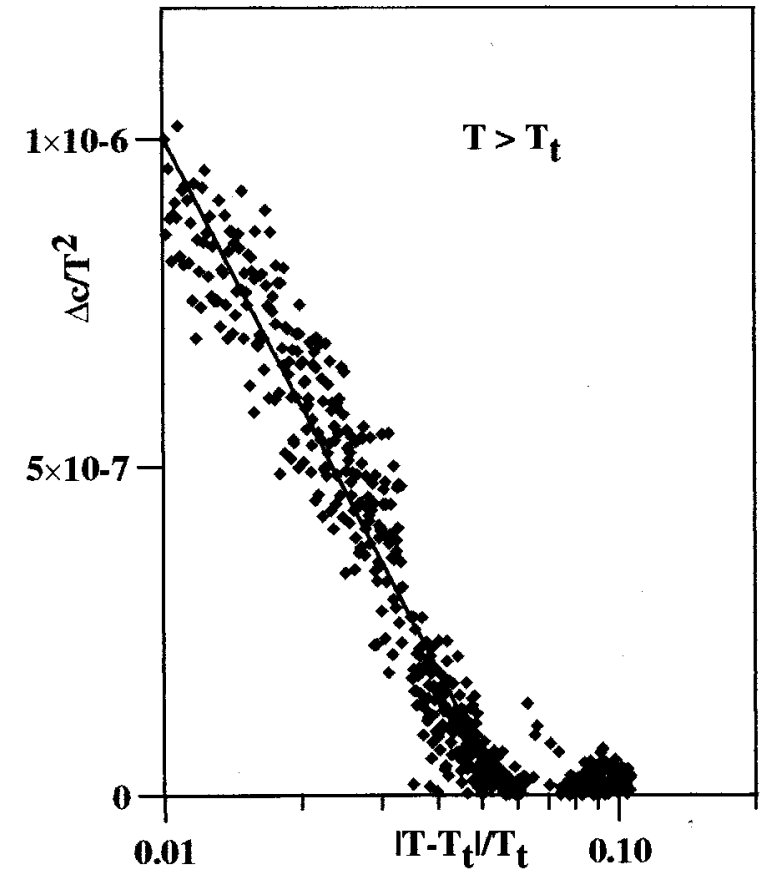

FIG. 5. Logarithmic temperature dependence of the singular part of DKDP specific heat above $T_{t}$.

this fluctuation with the acoustic mode component which is polarized along the ferroelectric axis. For uniaxial ferroelectric crystals, this singular part of the specific heat has a logarithmic divergence with temperature. Figure 5 presents, $\Delta c / T^{2}$ vs $\ln \left[\left(T-T_{t}\right) / T_{t}\right]$. Linear behavior is obtained similar to that found in $\mathrm{KDP}^{15}$ and $\mathrm{Pb}_{3}\left(\mathrm{PO}_{4}\right) .{ }^{16}$

Consider a 2-4-6 Landau Potential: ${ }^{18}$

$$
G(T, Q)=\frac{A}{2}\left(T-T_{c}\right) Q^{2}+\frac{B}{4} Q^{4}+\frac{C}{6} Q^{6},
$$

where $A, B, C$, and $T_{c}$, are constant and $Q$ is the order parameter. Below $T_{t}$ the singular part of the specific heat is expressed by:

$$
\Delta c=\frac{A^{2} T}{2 \sqrt{B^{2}-4 A C\left(T-T_{c}\right)}} .
$$

In Fig. $6(T / \Delta c)^{2}$ is represented versus $T-T_{t}$. The above expression is satisfied in the range of $4-15 \mathrm{~K}$ below $T_{t}$. The deviation from classical behavior near $T_{t}$ is attributed to the fluctuations of the order parameter and logarithmic behavior could be expected. Reese and May ${ }^{10}$ attempted to fit the singular part of the specific heat to a power law divergence and to a logarithmic divergence. Their data seem to show a better fit for a logarithmic divergence although their results were not completely conclusive. In Fig. 7 it is shown that $\Delta c$ linearly depends on $\ln \left(T-T_{t}\right)$ in a temperature range from $T_{t}$ to $1 \mathrm{~K}$ below $T_{t}$. These results confirm the logarithmic divergence suggested by Reese and May. Data obtained on heating are very similar to those obtained on cooling; nevertheless there are some differences we will discuss below. 


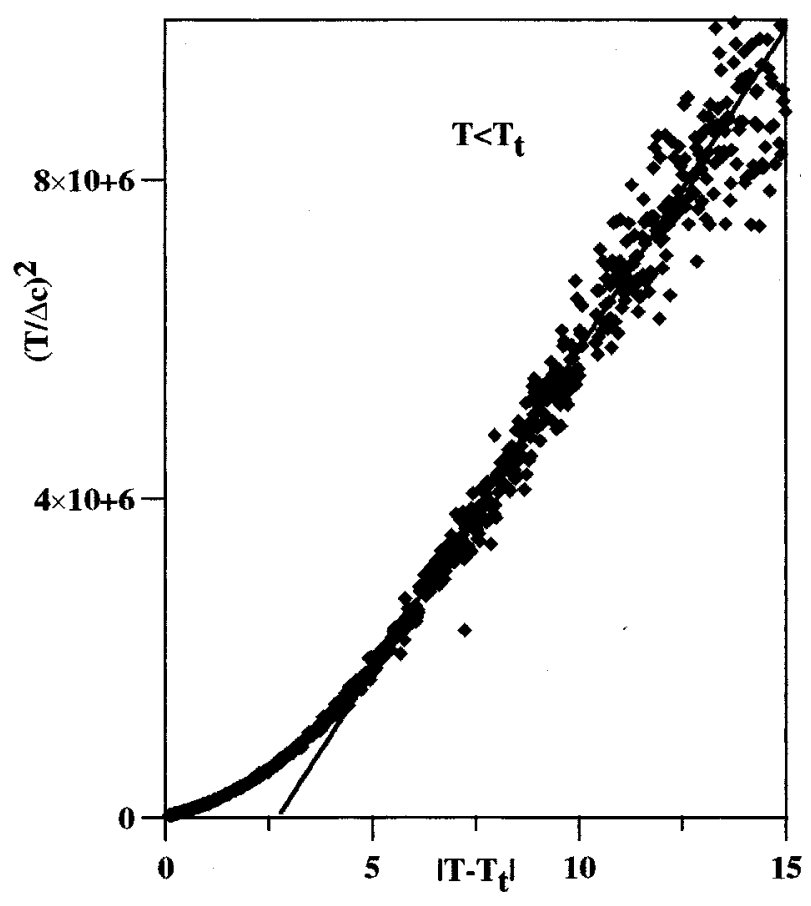

FIG. 6. Temperature dependence of the singular part of DKDP specific heat below $T_{t}$ according to Landau theory.

In Fig. 8, the specific heat and heat flux exchanged by the sample are represented versus block temperature. The transition occurs in a smaller temperature range than when cooling $(0.08 \mathrm{~K})$. As we would expect the heat power has the opposite sign as when cooling. During the transition the specific heat data show negative values. This behavior will be explained later.

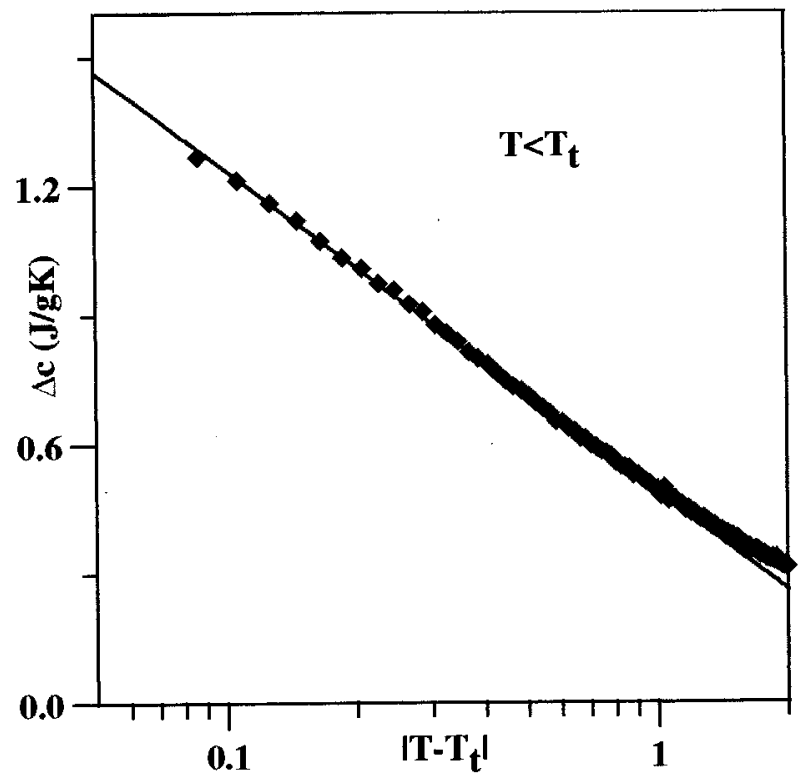

FIG. 7. Deviation from classical behavior: logarithmic temperature dependence of singular part of DKDP specific heat very close to $T_{t}$.

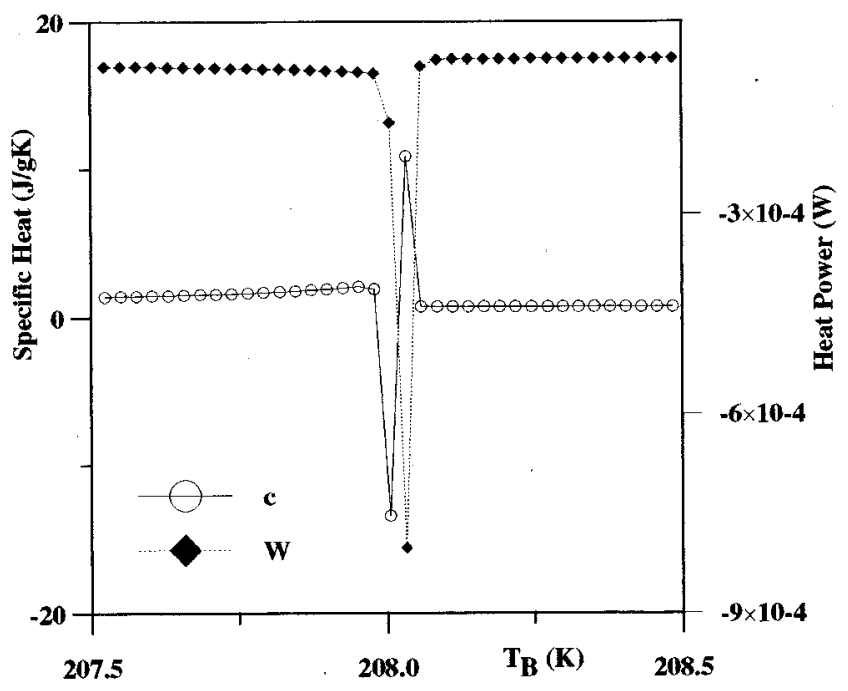

FIG. 8. DKDP specific heat $(\bigcirc)$ and heat power $(\diamond)$ vs block temperature during heating.

Both $T_{B}$ and $T_{S}$ are also shown in Fig. 3 on heating. Unlike when cooling, $T_{S}$ is not constant during the transition, but decreases to the constant value obtained on cooling. These results suggested we should carry out a more detailed study of the sample temperature evolution during the transition when heating and when cooling. This study will be discussed below.

Excluding data affected by the transition heat, the specific heat obtained when heating is also represented versus $T_{t}$ in Fig. 4. As is common in other ferroelectric materials the transition is broader on heating than on cooling and the tail on the paraelectric phase spreads over a larger temperature range $(\sim 30 \mathrm{~K})$. Data obtained during heating show similar behavior to those shown in Figs. 5, 6, and 7 .

To obtain more information about the sample temperature evolution during the transition, the emf $V_{1}$ (experimental zero) was measured every $15 \mathrm{~s}$ while cooling or heating the sample at the same constant rate of $0.06 \mathrm{~K} / \mathrm{h}$ but without dissipation in heaters $R_{1}$ and $R_{2}$. Figure 9 shows $W=\alpha V_{1}$ versus block temperature for both cooling and heating. On cooling, the transition spreads over a temperature range greater than on heating as we discussed above. We can also deduce that the kinetics are different: on cooling, different peaks appear which can be considered as consecutive partial changes of phases or rearrangement of domain structures. These peaks practically do not appear when heating. This behavior agrees with that reported by Bornarel and Cach. ${ }^{4}$ They measured the dielectric constant and simultaneously observed the ferroelectric domain structure of DKDP during the transition. On cooling from the paraelectric phase the domain structure exhibits rearrangement of the domain complexes and modification of the domain width with decreasing temperature. On the contrary, during heating the domain structure, which has been stabilized at low temperatures, does not change its configuration.

Figure 10 shows the evolution of the sample boundary temperature when cooling (a) and when heating (b). As was suggested above, when cooling $T_{S}$ seems to stay constant 


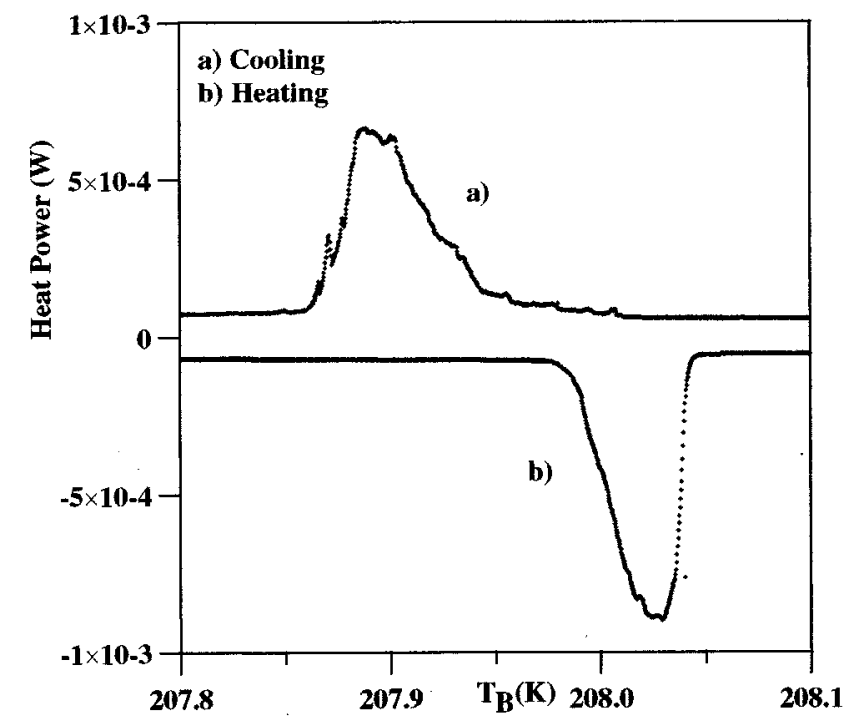

FIG. 9. Heat flux vs block temperature (a) when cooling and (b) when heating.

during the transition. From this data, together with the behavior shown in Fig. 9 we can deduce that this transition (cooling) is produced with equilibrium between para and ferroelectric phases.

On the contrary, as shown in Fig. 3, when heating a decrease of the temperature in the surroundings of the sample is produced. We must point out that the minimum value of $T_{S}$ in the transition region $(207.96 \mathrm{~K})$ practically coincides with the constant temperature of the transition (207.93 K) when cooling. These data suggest that the sample passes through metastable states and at a temperature higher that the transition temperature, a sudden phase transition occurs. Thus, the latent heat produces a temperature decrease at the sample boundary which is detected by the fluxmeters.

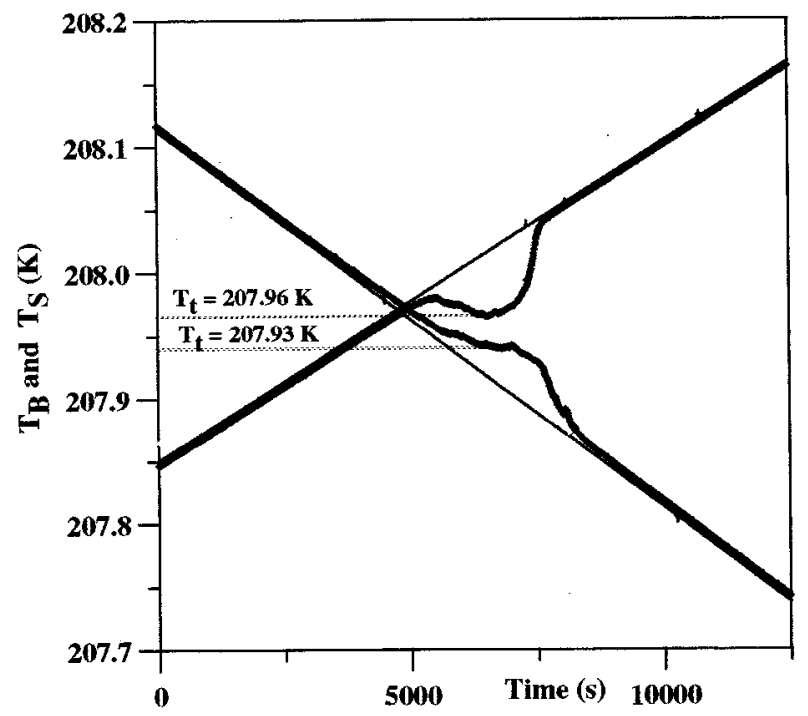

FIG. 10. Block temperature $T_{B}$ (thin line) and boundary sample temperature $T_{S}$ (points) vs time when cooling and when heating.
From the above we can deduce a very small thermal hysteresis which agrees with the behavior obtained by Bornarel and Cach. $^{4}$

On the other hand, integration of the heat power represented in Fig. 9 allows us to calculate the latent heat of this DKDP crystal. The estimated values are $2.32 \mathrm{~J} / \mathrm{g}$ and $2.20 \mathrm{~J} / \mathrm{g}$ for cooling and heating, respectively. These values are lower than the value of $2.99 \mathrm{~J} / \mathrm{g}$ obtained by Reese. ${ }^{10}$ The difference can be attributed to the fact that the sample studied in this article has a lower degree of deuteration than the sample studied by Reese whose transition temperature is higher than ours (220 and $208 \mathrm{~K}$, respectively). If we assume a linear relation between latent heat and deuteration degree, the above difference allows us to estimate that our sample is $77 \%$ deuterated; this value agrees well with that obtained $(82 \%)$ from the Brezina relation between transition temperature and degree of deuteration.

All of these measurements were also carried out with an applied constant stress of $\sigma=4.5 \pm 0.1$ bar on face (110). We obtained the same behavior described above $(\sigma=0.3$ bar $)$, even the same transition temperature. A $1 \%$ displacement of data was found, but this difference is similar to the error attributed to this type of measurement. This can be expected because Stasyuk et al. ${ }^{19}$ predict that only uniaxial pressure several orders of magnitude higher than applied during these measurements can have a significant influence on the specific heat of DKDP.

We must point out that uniaxial pressure similar to that applied in this study produces significant effects on the specific heat of $\mathrm{SrTiO}_{3}$ ferroelastic crystal. ${ }^{20}$ Nevertheless the coincidence of data obtained under 0.3 and $4.5 \pm 0.1$ bar uniaxial pressure can be considered as proof of the reproducibility of our measurements.

Now we will discuss the behavior of the specific heat data in the transition region. It has been shown that when cooling a double maximum appears (for both 0.3 and 4.5 \pm 0.1 bar) and when heating, negative values were obtained in both cases. These could be attributed to the fact that the change of the base line $V_{1}$ produces great errors in the determination of the integral $\mathrm{A}$ in Eq. (1), thus producing nonsense data. Nevertheless, we must point out that the variation of $V_{1}$ with time is taken into account in Eq. (1) and the method works properly when $V_{1}$ linearly changes with time. The time dependence of $V_{1}$ does not justify such big anomalies in the specific heat data. Thus, we think there must be another explanation for the above anomalous behavior of the data.

In a previous paper, the specific heat of a triglycine sulphate doped with L-alanine (LATGS) ferroelectric crystal was measured under an alternating electric field. ${ }^{21}$ Because of the decrease of bias and coercive fields near the transition temperature $T_{t}$, the sample carries out complete or minor hysteresis loops. Thus, in a temperature range near $T_{t}$, which depends on the amplitude and frequency of the applied field, the sample dissipates a heat power which depends on the temperature. In that study for an amplitude of $95 \mathrm{~V} / \mathrm{cm}$ and a frequency of $1 \mathrm{KHz}$ the temperature range where power was dissipated was $10 \mathrm{~K}$. This power $W$ was measured simultaneously with the "specific heat" of a steady state $\left(c^{*}\right)$. This 
"steady state specific heat" $c *$ is the relation between the heat exchanged by the sample between two steady states and the corresponding variation of the temperature at the sample surface. The heat exchanged by the sample as a consequence of the steady dissipation power is not taken into account. $c^{*}$ shows the following behavior: a decrease in the range where $\partial W / \partial T$ is negative and a large increase in the region where $\partial W / \partial T$ is positive.

To explain this effect the differential heat conduction equation of a solid with uniform heat power production dependent on the temperature, was studied. It was shown that the specific heat, $c^{*}$, of this dissipative medium is the equilibrium specific heat, $c$, (without dissipation) plus a term proportional to the derivative of the dissipative power with respect to the temperature. This statement was corroborated by experimental data.

In other words, in a dissipative medium there is an internal temperature distribution which depends on the dissipation power $W$. During the measurement process of the specific heat, the temperature of the sample changes. ${ }^{22}$ If $W$ depends on the temperature, the internal temperature distribution also changes. This change implies a supplementary term which must be added to the equilibrium specific heat. It was shown ${ }^{22}$ that this supplementary term is proportional to $\partial W / \partial T$.

A qualitative comparison between the behavior of DKDP and LATGS under dissipative conditions can be performed if we assume, in the first approximation, that on quasistatic cooling or heating the latent heat effect can be considered as an internal power source or power sink, respectively.

Although we do not have enough specific heat data (Figs. 2 and 8), when cooling it seems that the minimum between the two peaks appears at a temperature where the slope of $W$ is negative (negative contribution of the variation of the internal temperature distribution) while the second big maximum corresponds to the temperature where this slope is positive (positive contribution).

On heating we obtained similar behavior: a large decrease in the region where $\partial W / \partial T$ is negative, and an increase when $\partial W / \partial T$ is positive. When heating, the phase transition occurs through metastable states without equilibrium between phases. Thus we must expect a sudden change of enthalpy at these temperatures (a sharp heat power sink). Consequently, the negative contribution is so large that specific heat data reach negative value.
According to the above, we can explain the anomalous specific heat data, obtained during the transition, in terms of the change of temperature distribution inside the crystal produced by the latent heat.

We must conclude that when measuring specific heat near a first order transition it is necessary to take into account the influence of the latent heat on the measurement process. It is convenient to measure, simultaneously with the specific heat, other quantities which indicate clearly when the transition is produced. Thus, the technique of conduction calorimetry seems to be appropriate for this kind of study since it is capable of simultaneously measuring the specific heat and the heat flux exchanged by the sample. This information allows us to exclude data affected by the transition heat.

\section{ACKNOWLEDGMENT}

This work was supported by project PB91-601 of the DGICYT of Spain.

${ }^{1}$ Landott-Börnstein, Ferroelectrics and Related Substances: Non-Oxides, New Series (Springer, Berlin, 1982, 1990) Group III, vols. 16b, 28 b.

${ }^{2}$ V. H. Schmidt, A. B. Westrum, and A. G. Baker, Phys. Rev. Lett. 37, 839 (1976).

${ }^{3}$ B. Brezina, A. Fousková, and F. Smutný, Phys. Status Solidi 11, K149 (1972)

${ }^{4}$ J. Bornarel and R. Cach Ferroelectrics 124, 345 (1991).

${ }^{5}$ R. J. Nelmes, W. F. Kuhs, C. J. Howard, J. E. Tibballs, and T. W. Ryan, J. Phys. C 18, L1023 (1985).

${ }^{6}$ S. R. Andrews and R. A. Cowley, J. Phys. C 19, 615 (1986).

${ }^{7}$ E. V. Sidnenko and V. V. Gladkii, Sov. Phys. Crystallogr. 17, 861 (1973).

${ }^{8}$ W. Bantle, Helv. Phys. Acta 15, 373 (1942).

${ }^{9}$ B. A. Strukov, M. Amin, and V. A. Kopchik, Phys. Status Solidi 27, 741 (1968).

${ }^{10}$ W. Reese and L. F. May, Phys. Rev. 167, 504 (1968).

${ }^{11}$ W. Reese, Phys. Rev. 181, 905 (1969).

${ }^{12}$ M. C. Gallardo, J. Jiménez, and J. del Cerro, Rev. Sci. Instrum. 66, 5288 (1995).

${ }^{13}$ H. E. Buckly, Crystal Growth (Wiley, New York, 1951).

${ }^{14}$ J. del Cerro, J. Phys. E 20, 609 (1987).

${ }^{15}$ W. Reese and L. F. May, Phys. Rev. 162, 510 (1967).

${ }^{16}$ E. Salje and B. Wruck, Phys. Rev. B 28, 6510 (1983).

${ }^{17}$ H-J Bleif, R. A. Cowley, and R. J. Nelmes, J. Phys. C 15, 201 (1982).

${ }^{18}$ E. Salje, Phase Transitions in Ferroelastic and Co-elastic Crystals (Cambridge University, Cambridge, 1990).

${ }^{19}$ I. V. Stasyuk, I. R. Zachek, R. R. Levitskii, and K. V. Kukushkin (private communication), Preprint of VIII International Meeting on Ferroelectricity Gaithersburg, 1993.

${ }^{20}$ M. C. Gallardo, J. Jiménez, J. del Cerro, and E. K. H. Salje, J. Phys., Condens. Matter. 8, 83 (1996).

${ }^{21}$ J. del Cerro and S. Ramos, Ferroelectr. Lett. Sect. 16, 119 (1993).

${ }^{22}$ J. del Cerro and M. C. Gallardo, Ferroelectr. Lett. Sect. 18, 181 (1994). 